\title{
Immune System Process
}

National Cancer Institute

\section{Source}

National Cancer Institute. Immune System Process. NCI Thesaurus. Code C17937.

Any multicellular process of immune cells effecting or involved with the immune response

or immune maintenance. $(\mathrm{NCl})$ 